\title{
Driving Simulator Evaluation of the Failure of an Audio In-vehicle Warning for Railway Level Crossings
}

\author{
Grégoire S. Larue ${ }^{1,2} \cdot$ Christian Wullems ${ }^{1,3}$
}

Received: 10 April 2015/Revised: 2 May 2015/Accepted: 19 May 2015/Published online: 12 August 2015

(C) The Author(s) 2015. This article is published with open access at Springerlink.com

\begin{abstract}
It is impracticable to upgrade the 18,900 Australian passive crossings as such crossings are often located in remote areas, where power is lacking and with low road and rail traffic. The rail industry is interested in developing innovative in-vehicle technology interventions to warn motorists of approaching trains directly in their vehicles. The objective of this study was therefore to evaluate the benefits of the introduction of such technology. We evaluated the changes in driver performance once the technology is enabled and functioning correctly, as well as the effects of an unsafe failure of the technology? We conducted a driving simulator study where participants $(N=15)$ were familiarised with an in-vehicle audio warning for an extended period. After being familiarised with the system, the technology started failing, and we tested the reaction of drivers with a train approaching. This study has shown that with the traditional passive crossings with RX2 signage, the majority of drivers complied (70\%) and looked for trains on both sides of the rail track. With the introduction of the in-vehicle audio message, drivers did not approach crossings faster, did not reduce their safety margins and did not reduce their gaze towards the rail tracks. However, participants' compliance at the stop sign decreased by $16.5 \%$ with the technology
\end{abstract}

Grégoire S. Larue

g.larue@qut.edu.au

1 Centre for Accident Research and Road Safety - Queensland, Queensland University of Technology, Brisbane 4000, Australia

2 Australasian Centre for Rail Innovation, Canberra 2600, Australia

3 Cooperative Research Centre for Rail Innovation, Brisbane 4000, Australia

Editor: Marin Marinov installed in the vehicle. The effect of the failure of the invehicle audio warning technology showed that most participants did not experience difficulties in detecting the approaching train even though they did not receive any warning message. This showed that participants were still actively looking for trains with the system in their vehicle. However, two participants did not stop and one decided to beat the train when they did not receive the audio message, suggesting potential human factors issues to be considered with such technology.

Keywords Railway crossing $\cdot$ Safety $\cdot$ Intelligent transport systems $\cdot$ Compliance $\cdot$ Driving simulation

\section{Introduction}

There are currently 23,500 level crossings in Australia [11], broadly divided into one of two categories: active level crossings with flashing lights; and passive level crossings controlling traffic solely with stop and give way signs. Crashes at level crossings continue to result in significant human and financial cost to society. According to the ATSB, there were 601 road vehicle collisions at Railway Level crossing (RLX) between July 2002 and June 2012 [1]. Many of these collisions between road vehicles and trains occur at level crossings with passive controls.

Closure of crossings, grade separation and installation of active protection at level crossings with passive controls are undoubtedly the most effective approaches to reducing the risk of collisions at railway level crossings. However, the feasibility of such approaches is questionable given economic and logistical implications [4]: it is impracticable to upgrade the 18,900 passive crossings due to various challenges such as the high number of private and 
occupational crossings $(13,000)$, the remote location nature of such crossings, the lack of power available on site and the difficulty to reach viable cost-benefit ratios for crossings with low road and rail traffic.

Several analyses have demonstrated that errors or violations on the part of the road user represent the largest contributor to RLX crashes [1, 12, 19], indicating the need for innovative interventions targeted at drivers to complement current railway interventions. The rail industry is therefore interested in new approaches to reduce the number of crashes at passive level crossings. One of them is to use Vehicle to Infrastructure (V2I) and Vehicle to Vehicle (V2V) communications to warn drivers about the approach of a train directly in their vehicles. Significant research has been conducted to study driver behaviour and responses to various traditional road-based interventions at different types of RLX. This resulted in some evidence to suggest the effectiveness of traditional approaches to level crossing safety, however, much more research is required to properly evaluate emerging technologies. Emerging technologies might be optimally used as a comparatively low-cost approach to increasing safety at passively protected crossings.

While in-vehicle technologies helping drivers perform the driving task become more pervasive, there is a lack of evaluation of the effects such interventions would have on driver behaviour if applied to railway crossings, particularly in the event of an unsafe failure of the technology [19].

When evaluating an intervention at railway crossings such as an in-vehicle Intelligent Transport System, it is important to ensure that such systems are efficient in attracting drivers' attention and ensure compliance [16, 19]. In Australia, passive crossings with 'Stop' signs require the driver to completely stop their vehicle at the appropriate road marking and to look for the presence of a train in both directions. If a train is entering the railway crossing or an approaching train is visible from the crossing, the driver is expected to give way to the train. After the train has cleared the crossing, and no other trains are approaching, the driver may traverse the crossing. Also, such systems must be developed to avoid over-reliance and subsequent reductions in the performance of protective crossing behaviours, such as stopping compliance rate, monitoring approach speeds and scanning the crossing environment [2]. Indeed, familiarity with a level crossing has been shown to reduce the perception of risk, and encourage drivers to engage in risk-taking behaviours. This is particularly the case for passively protected crossings, where regular road users encounter trains infrequently and tend to know the train timetables [3], leading to reduced number of visual checks (a factor which was attributed to one of the fatalities analysed in [18] ).

Various studies have been conducted in order to evaluate the effect of various railway level crossings interventions on driver behaviour, such as traffic lights [9], rumble strips and in-vehicle systems [6, 17]. Traffic signals at railway level crossings do not appear to offer any safety benefits over and above flashing red lights, and rumble strips seem to be effective in reducing approach speed but not compliance at passive crossings. On the other hand, invehicle interventions tend to result in driver behaviour similar to active crossings, which result in higher compliance when trains are approaching railway crossings. Among in-vehicle interventions, an audio message has been shown to be more effective than visual information on a GPS-like device [6]. With an audio message, drivers tended to use the in-vehicle warning as assistive information, while using visual feedback resulted in drivers using the in-vehicle system as the main control of the crossing, disregarding the STOP sign present at the crossing. Research has also shown that drivers exposed to in-vehicle warning system for railway crossings held a strong intention to use such technology if available, particularly in the context of passive crossings. Such positive intention was largely explained by the fact that such technology was perceived to be useful, easy to use and socially acceptable [7].

Such studies provide a good rationale for the development of in-vehicle interventions, but they are lacking an evaluation of the potential familiarisation of road users with the technology, which could then result in driver complacency. It is the aim of this study, which familiarises participants with a new audio in-vehicle technology for an extended period of time, and then trials an unsafe failure of the technology.

The objective of this paper is to provide a human factors study evaluating operational performance of an audio invehicle message warning participants about approaching trains at railway level crossings using a driving simulator. In particular, this study will evaluate driver behaviour in case the technology fails to provide warning of the approaching train. A simulation-based study was conducted to address these questions through the development of specific scenarios in a controlled simulation environment. This approach allowed evaluation of the following research questions with statistical power:

- What is the current performance of drivers at railway level crossings with passive controls (RX2)?

- What changes are evident in driver performance once the technology is enabled and functioning correctly?

- What is the road user performance when the audio ITS does not provide the warning message about the approach of a train (failure mode of the technology) while approaching a level crossing.

This was done through the observation of changes of driver behaviour in terms of compliance, gaze patterns, 
approach speed and safety distances. Questionnaires were also used to obtain feedback from drivers after they trialled the technology.

\section{Experiment}

\subsection{Trialled In-vehicle Intervention}

The audio in-vehicle warning system implemented in this study used the speakers of the simulator positioned inside the vehicle. When the driver was within 200 metres of the crossing and a train was within $20 \mathrm{~s}$ of the crossing, which is the minimum warning time required in the Australian standard (AS1742.7) for single track crossing [15]. At activation, the system provides the following audio stimuli:

- The sound of a warning bell similar to the bell used at Australian railway level crossings; followed by

- A spoken message: "Warning. Train at crossing. Prepare to stop.".

This sequence was repeated up to three times until the speed of the driver was below 20 kilometres per hour.

\subsection{Experimental Design}

This study was designed to assess the changes to road user behaviour when the warning fails and the RX2 (passive level crossing control with stop sign and cross-bucks) is the primary control at a level crossing. The familiarisation phases were designed to enable participants to initially become accustomed to the simulator and to provide them with an environment in which they are exposed to RX2 crossings before and after the in-vehicle warning system was operational. Participants were only told that the vehicle was fitted with an in-vehicle system providing audio messages when they were required to stop at a crossing due to the presence of a train. Data were not analysed during these phases.

A within-subjects design was used in order to evaluate the effects of familiarisation on driver decisions with the technology. The study was composed of two sessions, which were composed of 12 and 13 repetitions of a similar scenario respectively, varying in terms of presence of the technology and presence of a train. The crossing with train approaching was varied between subjects. Details of these scenarios are provided in Table 1 for the first session and Table 2 for the second session. The first half of the first session was a baseline of driver behaviour at traditional passive crossings with a Stop sign. The second half was used to introduce participants with the operational in-vehicle technology. The second session was also using the audio in-vehicle warning. This session focused on creating a habit where the technology worked properly, until it failed to provide the warning message as a train was approaching (trial 2e) in a way that the driver could not be aware of the failure until they saw the train (unsafe failure of the system).The technology started failing in the middle of the second session, but the participant could not have noticed the change, as no train was approaching crossings. During the last trial, the train was programmed to arrive at the crossing $12 \mathrm{~s}$ after the driver reached a full stop at the level crossing.

This type of study had to be implemented in a driving simulator, as the effects of unsafe failures of the technology were investigated with participants. On-road evaluation would not be possible due to ethical and safety issues. The rural level crossing located on Lane road, Lanefield, Australia was replicated in the driving simulator environment (see Fig. 1).

A power analysis was used to determine the number of participants required for this study. The required sample size $\mathrm{N}$ to obtain a significant result at level $\alpha=0.05$ for a power $\beta$ when comparing the mean of a variable of interest at railway crossings with and without the intervention is given by [5], assuming similar variance

$N=\frac{2(u+v)^{2}}{d^{2}}$

where $u+v=2.8$ for $\beta=0.8$ and $u+v=3.24$ for $\beta=0.9$ and $d=\left(\mu_{1}-\mu_{0}\right) / \sigma_{0}$ is the standardised mean difference.

For detecting high size effects $(d>1), 16$ participants are required at power $\beta=0.8$ and 21 participants are required for a power $\beta=0.9$.

\subsection{Participants}

Twenty subjects volunteered to participate in this study. Five subjects ( 4 females and 1 male) were not able to complete the study due to motion sickness in the driving simulator, resulting in a sample size of 15 participants (11 males and 4 females) aged between 27 and 53 (mean age $=37.5$ years, $\mathrm{SD}=7.0$ ). Participants were recruited from the Queensland University of Technology (students and staff members) as well as from the general public. Participants had an open Australian driving licence. All subjects provided written consent for this study, which was approved by the Queensland University of Technology ethics committee. Participants were paid AUD \$100 for completing the two driving sessions; and AUD \$20 in case they were not able to complete the study. 
Table 1 Design of the first session
Session 1: RX2 and ITS

1a. Familiarisation ( $3 \mathrm{RX} 2$ crossings and one train at crossing)

1b. Five trials with $3 \mathrm{RX} 2$ crossings and no train approaching

1c. Familiarisation with $3 \mathrm{RX} 2$ crossings with audio ITS and one train at crossing

1d. Five trials with 3 RX2 crossings with audio ITS and no train approaching

Table 2 Design of the second session

Session 2: RX2 and ITS (partial coverage/failure)

2a. One trial with $3 \mathrm{RX} 2$ crossings with audio ITS and one train at crossing

2b. Five trials with $3 \mathrm{RX} 2$ crossings with audio ITS and no train approaching

2c. One trial with $3 \mathrm{RX} 2$ crossings with audio ITS and one train at crossing

2 d. Five trials with $3 \mathrm{RX} 2$ crossings with the audio ITS failing and no train approaching

2e. One trial with 2 RX2 with the audio ITS failing, no train approaching. A third RX2 with the audio ITS failing and a train approaching

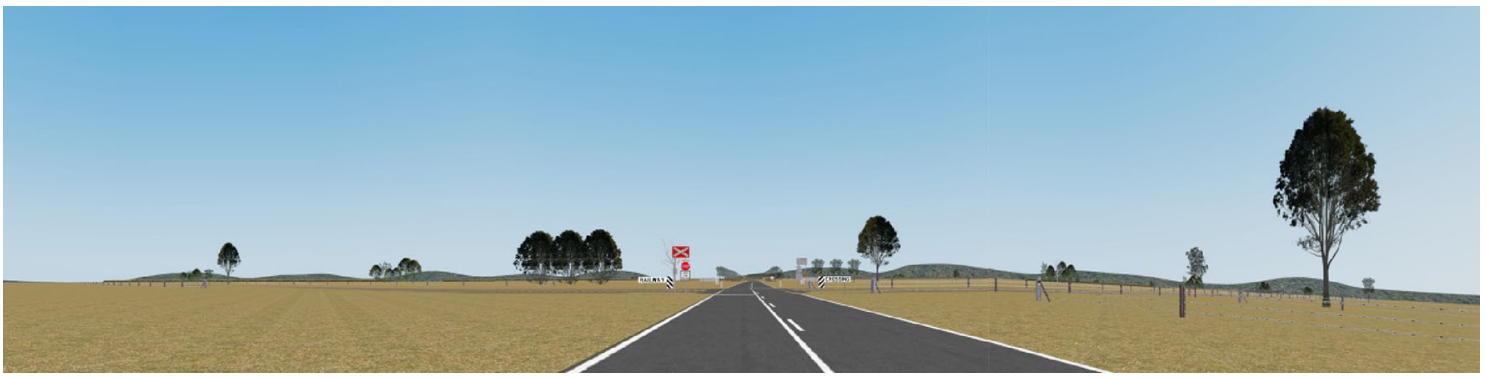

Fig. 1 Typical Australian passive crossing as implemented in the simulator

\subsection{Ethical Clearance}

Ethical clearance for this research was obtained from the QUT Human Research Ethics Committee (reference number 1300000298).

\subsection{Procedure}

Each participant took part in two sessions taking approximately $2 \mathrm{~h}$ each. During each session, the participant was performing a repetitive succession of simulated driving tasks consisting of driving an itinerary from start to end. The itinerary was a typical rural Australian road, with 3 passive railway level crossings with stop signs and two road intersections (one with a stop sign, one with right-ofway).

Upon arrival, participants provided written consent to participate in the study, and were then provided with a short familiarisation drive in the simulator allowing them to become accustomed to accelerating, stopping and driving though intersections, passive railway crossings and curves.
In the middle of the first session, the audio system was implemented in the driving simulator, and the system was presented to the participants. The message was played to the participant, and they were then given a familiarisation drive with the ITS switched on to enable them to feel confident whilst driving with the system activated. Participants subsequently drove with the system active until the end of both sessions.

The second session took place on another day, or a couple of hours after the participant finished the first session. At the start of the second session, the participant was reminded that the car was equipped with a system giving audio messages when trains are approaching a level crossing.

While participants were driving, a researcher was manually recording head movements as drivers were driving toward railway crossings. The researcher had a table where he could tick the following categories: both sides checked, only one side (left or right) checked and no sides checked. From these observations, compliance was defined as checking both sides of the crossing before going through the crossing. 
Surveys were administered post-trials for the human factors evaluation study. The questionnaires assessed driver demographics, general driving experience and exposure to passive crossings in Australia, and experience of the technology in accordance with the Technology Acceptance Model. They were administered to participants directly as a paper version at the end of the second session. Questionnaires took 10-15 min to complete.

\subsection{Materials}

\subsubsection{Driving Simulator}

The study was conducted on CARRS-Q's Advanced Driving Simulator (see Fig. 2). The simulator included a complete Holden Commodore vehicle with automatic transmission, working controls and instruments. The simulator is based on $\mathrm{SCANeR}^{\mathrm{TM}}$ studio software (Oktal) with eight computers, three projectors and a six degree of freedom motion platform. When seated in the simulator vehicle, the driver is immersed in a virtual environment which includes a 180 degree front field view composed of three screens, simulated rear view mirror images on LCD screens, surround sound for engine and environment noise, real car cabin and simulated vehicle motion. The road and surrounding environment were designed to represent, as closely as possible, a rural railway crossing in accordance with Australian Standards at railway crossings (see Fig. 1).

\subsubsection{Questionnaires}

A general questionnaire was first given to participants to cover general demographics such as age, gender and driving experience, with particular focus on railway level crossings (with a distinction between active and passive crossings). Experience of incidents at railway crossings in

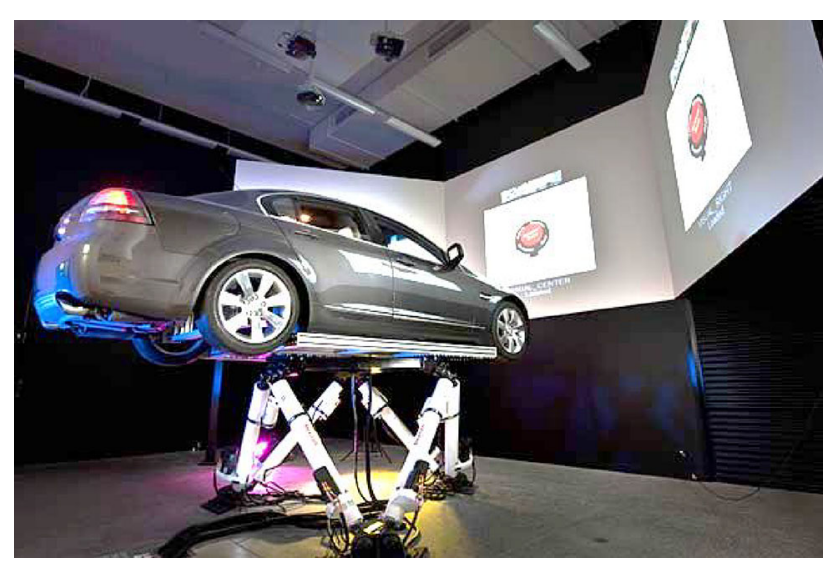

Fig. 2 CARRS-Q's advanced driving simulator the last 5 years was also investigated. Questions gauging such experience included

- You thought there was no train approaching the crossing, but when you could not stop anymore, you realised a train was approaching.

- The signal at the crossing was off, but when you could not stop anymore, the signal started flashing.

- You got stuck on the crossing because of the traffic in front of you.

- You had a collision with a boom gate.

- Other. Please describe.

As this study focuses on malfunctions of technology at level crossings, participants were also asked about their perception of likelihood of signal failure at crossings; their expected behaviour if the flashing lights were always on and no train was coming or if the lights were always off, even if a train was approaching. Participants were also asked about their perception of the issues at level crossings, and how predictable are trains arriving at crossings in their area. Knowing the times when trains are present at crossings is a known contributing factor to crashes at crossings, which can also be correlated with their perception of the usefulness of the system.

Questions were then presented about the technology trialled. It focused on the Technology Acceptance Model, which targeted the perceived usefulness and the perceived ease of use of the technology. Perceptions of advantages and disadvantages of the system were also investigated. The failure of the system was also of interest in this questionnaire, with questions such as: How often would this system have to fail for you to consider not useful anymore?

We also asked to what extent the system would change their behaviour, as participants trialled the system and should have a good idea of the potential effects of such system on their driving behaviour. Changes in their perception of system usefulness after they saw it failing were also investigated.

\subsubsection{Software}

Data analyses were conducted with the statistical package $\mathrm{R}$, version 3.1.1.

\section{Data Analysis}

Data analysis was performed on data collected from the advanced driving simulator as well as surveys conducted after the simulator study.

The simulator study comprised a baseline data collection with traditional RX2 signage at the railway crossings, followed by the introduction of a new audio in-vehicle technology designed for railway level crossings. After a 
familiarisation with the technology, the failure of the technology was trialled during the last run of the second driving session. The driving simulation provided a controlled environment that allowed participants to experience the system working correctly, familiarise themselves with the system and then facilitated assessment of their behaviour once the system was failing.

The dependent variables under investigation in this data analysis were the following:

- The variation of the approach speed when no train was approaching the crossing. By comparing to the baseline, the perception of the level crossing as being actively or passively protected can be inferred. For the driving simulator, speed profile can be measured at any distance to the crossing, and speed 20 and $40 \mathrm{~m}$ away from the crossing were investigated, as they have been shown by various studies in the literature to be distances where speed varies for passive crossings. Speed $150 \mathrm{~m}$ to the crossing was also investigated in order to ensure that no differences were present before approaching the crossing;

- Compliance with the crossing signage;

- Gaze behaviour when participants approach a railway crossing;

- Safety distances: reaction time distance, minimum possible braking distance and safety margin, as defined in [8] and

- Subjects' perceptions of the systems from the surveys.

The analysis evaluated how the following independent variables (type of signal at the RLX) had effects on the dependent variables presented above:

- RX2 with no train;

- RX2 with the in-vehicle audio warning with no train and

- RX2 with the in-vehicle audio warning failing while a train is approaching the crossing

Statistical analyses were conducted to evaluate the effects of the failure of the technology. Generalised Linear Mixed Modelling was used in order to take into account the repeated measures study design.

\section{Results}

\subsection{Driving Simulation}

\subsubsection{Observed Driving Behaviours}

The following behaviours have been observed in the driving simulator when the audio in-vehicle message failed to be played by the vehicle:
- Stopped without any issues (12 participants);

- Decided to beat the train (1 participant);

- Did not change their behaviour and did not stop at the crossing, without any indication as to whether they realised a train was approaching with the in-vehicle system failing (2 participants).

With the participants completing the study, we obtained $80 \%$ of participants stopping without any problems when the system failed to provide the warning message; and $7 \%$ saw the train and decided to beat it. It was not possible to ascertain the behaviour of $13 \%$ of participants as they were not complying before the occurrence of the failure.

\subsubsection{Effects on Approach Speed}

Participants had a similar approach $150 \mathrm{~m}$ to the crossing. The in-vehicle technology is used only to increase awareness of drivers while approaching crossings, and participants were expected to stop the same way as for the RX2 signage. Speed closer to the crossing were very similar with the ITS system implemented, whether it was working or failing with a train approaching (see Table 3).

The average speed $150 \mathrm{~m}$ to the crossing was $72.5 \mathrm{kph}$ during the baseline with RX2 signage. No differences were observed with the in-vehicle warning provided. It has also to be noted that speed increased with the number of trials in a parabolic manner with a factor $.02(p<.001)$. This means that on average, participants speed increased by $2.9 \mathrm{kph}$ by the time they reached the last trial run.

The same trend was observed 40 and $20 \mathrm{~m}$ to the crossing. At these locations, speed was on average 33.8 and $19.3 \mathrm{kph}$, respectively. Such value held with or without the technology. Speed also increased with the number of runs in a parabolic manner with factor $.02(p<.001)$.

\subsubsection{Effects on Stopping Compliance}

The stopping probability observed in the driving simulator for the different sign conditions is reported in Table 4. On average probability of stopping with the RX2 signage was $69.8 \%$. Statistical analyses for repeated measures were conducted and showed that the probability of stopping changed with the introduction of the in-vehicle audio warning. It decreased to $53.4 \%(p<.001)$ showing that participants started to rely on the technology for their decision-making in this mode of operation. While participant compliance reduced with the technology, the proportion of participants stopping at the crossing with the train approaching and the in-vehicle system not working was very high $(80 \%)$ and suggest that participants were still actively analysing the situation at the crossing with this technology installed (at least for the time involved in this trial; this could 
Table 3 Approach speeds (kph, standard deviations in brackets)

\begin{tabular}{llll}
\hline Level crossing & $150 \mathrm{~m}$ & $40 \mathrm{~m}$ & $20 \mathrm{~m}$ \\
\hline RX2, no train & $71.3(7.4)$ & $33.9(13.2)$ & $18.4(16.6)$ \\
RX2, ITS, no train & $72.9(9.7)$ & $34.6(12.9)$ & $18.9(15.7)$ \\
RX2, failing ITS, train approaching & $71.2(9.8)$ & $32.5(16.8)$ & $21.2(19.5)$ \\
\hline
\end{tabular}

Table 4 Stopping probability (standard deviation in brackets)

\begin{tabular}{ll}
\hline Level crossing & Stopping probability \\
\hline RX2, no train & $69.8 \%(.46)$ \\
RX2, ITS, no train & $53.4 \%(.48)$ \\
RX2, failing ITS, train approaching & $80.0 \%(.38)$ \\
\hline
\end{tabular}

change with familiarity with the system over longer periods of time). We did not observe any participants failing to detect the train with the system failing.

\subsubsection{Effects on Head Movements Toward Rail Tracks}

Rates of gaze compliance in the different conditions are reported in Table 5. Statistical analyses (taking into account repeated measures from the same participants) showed that participants checked on average both sides of the rail track $96.9 \%$ of the time, independently of the factors under investigation in this study. No statistically significant differences were observed with the technology in place.

\subsubsection{Effects on Safety Distance}

Reaction time distance, minimum braking distance and safety margins in the different conditions are reported in Table 6. Statistical analyses (taking into account repeated measures from the same participants) showed that all of the factors under investigation in this study had no statistical effect on these variables (Fig. 3).

\subsection{Feedback from Drivers}

\subsubsection{Positive Feedback}

Participants were asked about the advantages they saw in using such an in-vehicle system, and their feedback is presented in the following subsections.

Table 5 Compliance in gaze behaviour

\begin{tabular}{ll}
\hline Level crossing & Compliance \\
\hline RX2, no train & $100 \%(-)$ \\
RX2, ITS, no train & $96.5 \%(.18)$ \\
RX2, failing ITS, train approaching & $93.3 \%(.26)$ \\
\hline
\end{tabular}

4.2.1.1 Additional Support as a Complementary System The advantage most reported by participants (6 participants) is that this system can be used as a backup system, which would support drivers approaching and traversing crossings. One male participant (46 years old) reported that this system acts as a backup system for events where driver inattention or drowsiness has the potential to result in a collision with a train. A female (29 years old), reported that this system is a safety net for driver distraction, while another one (53 years old) said that it is reinforcement; the two together would probably help. However, failure rate would have to be nil.

4.2.1.2 Better Awareness at the Crossing and Better Visibility of the Crossing Five participants felt that this technology would increase their awareness when approaching a crossing. A male, 36, reported that this equipment is certainly useful in providing awareness amongst drivers who are paying less than adequate attention, or drivers who are fatigued. Another one (27 years old) wrote that it provides an additional cue and hence improves safety. A female participant (34 years old) added that this system made her aware of train crossing and made her more careful when crossing train track.

Participants also reported potential positive effects in case visibility is reduced while approaching the crossing, such as in night time conditions: if it works, it's a helpful aid particularly at night time when vision may be impaired if passive crossing.

4.2.1.3 Mitigation of Driver Inattention This system is particularly perceived as having benefits for inattentive drivers, whether due to sleepiness or distractions. It is useful for drivers who may be tired or are momentarily inattentive. It could save their life if they miss the stop sign (male, 43). A female participant (42) reported that this additional audio warning is harder to ignore even if your eyes are heavy. It was perceived as very useful if integrated within the car: This may help some people, especially if there are other distractions in the car. This should be built into the radio in the car so it temporarily mutes the music to make it very obvious (male, 35).

4.2.1.4 Ease of Use It was perceived as an easy system to use, which could be useful in rural areas rather than metropolitan areas where railway crossings are already 
Table 6 Safety distances (standard deviation in brackets)

\begin{tabular}{llll}
\hline Level crossing & Reaction time distance & Minimum braking distance & Safety margin \\
\hline RX2, no train & $88(54)$ & $28(7)$ & $130(36)$ \\
RX2, ITS, no train & $92(53)$ & $29(7)$ & $124(35)$ \\
RX2, failing ITS, train approaching & $84(29)$ & $31(14)$ & $132(39)$ \\
\hline
\end{tabular}

Fig. 3 Effects of the technology on safety distances

\section{Effects of the technology on safety} distances

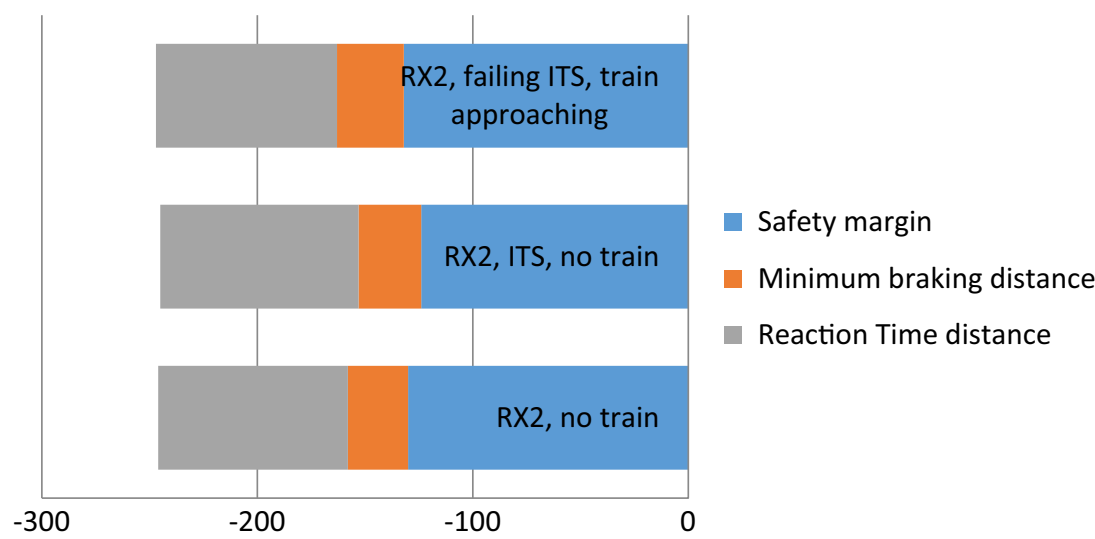

equipped with flashing lights and boom gates. It was perceived useful particularly if it was integrated into other technological aids already being used, such as GPS (male, 36).

\subsubsection{Negative Feedback}

After experiencing the failure of the technology, participants also reported a number of potential issues with such systems.

4.2.2.1 Facilitating Complacency The highest concern was related to complacency, with 10 participants saying that such system could lead to complacency: many people may depend on the technology and fail to stop or may not stop on time when a train is actually coming and the technology failed to activate. Participants felts that drivers may rely too much on the system and not use their eyes and ears for situational awareness, which highlights the potential risk of reduction of active checks while approaching a crossing, once used to having a reliable warning message in the vehicle. One female participant reported that drivers could become reliant on it and less attentive to visual warnings such as signs, road markings, etc. It could result in a tendency to perhaps not stop at a crossing, despite signs, if the alarm does not sound (male, 36). Implementing such technology could encourage people to rely on it which could be dangerous if it fails (male,
42), which could even make crashes happen more often (male, 32).

4.2.2.2 Cost Participants reported that this system should only be considered as a backup system that should only complement traditional solutions in place such as lights or stop sign (male, 43). However, the risk that drivers use this system as the primary control when approaching the crossing suggests that such technologies would be a good initiative if it works $99.99 \%$ (at least) $^{1}$ of the time and if it fails in a safe manner, as for flashing lights installed at level crossings. This raises concerns about the cost such technology would have on the driver, as the cost to install such system in a car could be expensive, and could require maintenance and repairs by the owner.

4.2.2.3 Potential Failures Experiencing the failure of the technology in the last trial resulted a decrease in the perception of the usefulness of the technology for $47 \%$ of participants, which shows the need for a reliable system which would fail in a safe manner.

4.2.2.4 Annoyance This device could also annoy drivers if it was activated quite often: it will become so annoying if the warning comes up too often (male, 36).

\footnotetext{
$\overline{1}$ This is only opinion from the participant. Safety reliability analysis is required to determine how reliable equipment needs to be.
} 


\section{Discussion}

This driving simulator study has shown that with the traditional passive crossings with RX2 signage, the majority of drivers complied (70 \%) by stopping at the crossing and all participants looked for trains on both sides of the rail track. A high number of participants (30\%) did not comply at passive crossing, and this is consistent with observations made at the Lane road, Lanefield crossing, which the scenarios in the driving simulator were based on. This can largely be explained by familiarity with the crossing and the high visibility that is characteristic of this crossing (sighting distance above $1.5 \mathrm{~km}$ in each direction).

With the introduction of the audio in-vehicle warning for railway level crossings, no differences were observed for approach speeds, gaze behaviour and safety margins. Drivers did not approach crossings faster and did not reduce their gaze towards the rail tracks. However, participant compliance at the stop sign decreased with the technology installed in the vehicle: stopping compliance reduced to $53.5 \%$, which is a $16.5 \%$ reduction in stopping compliance.

The effect of the failure of in-vehicle audio warning was also assessed with the simulator study. It showed that most participants did not experience difficulties in detecting the approaching train even though they did not receive any warning message. This showed that participants were still actively looking for trains with the system in their vehicle. Most participants stopped, but two participants did not (it was their usual behaviour at the crossing without trains after familiarisation) and one decided to beat the train. Such behaviour suggests that these participants might have been re-enforced in their decision to go through the crossing by the lack of message from the system. Two of these three participants have interpreted that it was safe to cross, as they later reported that they did not notice that the technology had failed. However, if the crossing was actively protected, it would have been closed (flashing lights). Further, drivers are required by law to not proceed through level crossings when a train is visible. This highlights the need for such technology to be failsafe.

The characteristics of the level crossing in terms of sighting distance made it easy for participants to see the train. Further research should be conducted in conditions where the train could be seen only when closer to the crossing (by reducing the sighting distance to the minimum distance required in the Australian standard). Further, this study induced a certain level of familiarity with driving through crossings with the technology, using two repetitive sessions. This can be confirmed by the reduction in stopping compliance, which suggests that drivers started to rely on the technology. However, the study was of a limited time, and effects on a longer term could not be estimated. The reduction of compliance at the crossing with the introduction of the technology as well as the feedback obtained from participants at the end of their trial suggest that with time drivers may start to largely rely on the technology if they perceive it reliable. This could potentially reduce the ability of drivers to detect approaching trains when the system fails. The decrement of compliance with the introduction of the technology should be carefully taken into consideration if such system was to be implemented at crossings. Reliability and integrity of the technology should be analysed and used to model risks involved with the implementation of such technology. In particular, overall effects on safety at each individual crossing should be evaluated in order to ensure that risk does not increase.

This study also suggests that in the long term, there could be negative effects on driver performance of introducing such technology. Hence the system needs to be able to manifest its failure modes to the driver in a clear manner. This could be mitigated by providing drivers with interfaces that display contextual information about the reliability of the systems, as it has been shown that complacency becomes less likely when information about the reliability of the instructions is provided [13]. Various methods have shown promising results for reducing complacency, such as displaying dynamically the system's confidence in its recommendations [10] or making the automation failure more salient [14]. Other human factor issues, such as the effects the availability of the system at some crossings and not others, while outside the scope of this study, should be considered if such device was to be implemented.

\section{Conclusion}

This driving simulator study has shown that the current performance of driver behaviour at traditional passive crossing with a stop sign is low after being familiarised with a crossing with low train traffic and with high sighting distances. This is similar to the behaviour that can be seen on roads with this type of level crossings. The introduction of the audio in-vehicle warning resulted in a reduction of stopping compliance at the stop sign, suggesting that drivers tend to use such system not only as an assistive device but more as a primary control. The warning device has no significant effect on gaze behaviour, approach speeds or safety distances. Participants were still actively checking the rail environment in order to detect trains, and drivers were all able to detect the approaching train when the invehicle system was failing unsafely. However, two participants did not stop and one decided to beat the train 
when they did not receive the audio message. Two of these participants did not realise the technology was failing, and they decided to proceed through the crossing, as the technology suggested it was safe to do so. This study has highlighted potential complacency issues with the introduction of new in-vehicle technologies for railway level crossings, both with the driving performance observed after familiarisation with the crossing, and self-reports from participants at the end of the driving task. This shows the need for taking into consideration the failure modes of such in-vehicle systems during the engineering design phases and also design how to provide this information to the road users.

Conflict of interest The authors declare that they have no conflict of interest.

Open Access This article is distributed under the terms of the Creative Commons Attribution 4.0 International License (http://crea tivecommons.org/licenses/by/4.0/), which permits unrestricted use, distribution, and reproduction in any medium, provided you give appropriate credit to the original author(s) and the source, provide a link to the Creative Commons license, and indicate if changes were made.

\section{References}

1. Australian Transport Safety Bureau (2012) Australian rail safety occurrence Data 1 July 2002 to 30 June 2012. In: Australian Transport Safety Bureau AG (ed) Australian Transport Safety Bureau. Statistical Publication Investigation number: RR-2012010

2. Caird JK, Creaser JI, Edwards CJ, Dewar RE (2002) A human factors analysis of highway-railway grade crossing accidents in Canada. Transp Canada, Canada

3. Caird JK, Smiley A, Fern L, Robinson J (2011) Driving simulation design and evaluation of highway-railway grade and transit crossings. In: Fisher DL, Rizzo M, Caird JK, Lee JD (eds) Handbook of driving simulation for engineering, medicine, and psychology. CRC Press, Hoboken
4. Cairney P (2003) Prospects for improving the conspicuity of trains at passive railway Crossings. Road safety research report. Australian Transport Safety Board, Vermont South

5. Kirkwood BR, Sterne JAC (2003) Essential medical statistics. Blackwell Science, Malden

6. Larue GS, Kim I, Buckley L, Rakotonirainy A, Haworth NL, Ferreira L (2014) Evaluation of emerging Intelligent Transport Systems to improve safety on level crossings-an overview. In: 2014 Global level crossing symposium, 4-8 August 2014, Urbana

7. Larue GS, Rakotonirainy A, Haworth NL, Darvell M (2015) Assessing driver acceptance of Intelligent Transport Systems in the context of railway level crossings. Transp Res F 30:1-13

8. Lay MG (2009) Handbook of road technology. Spon, New York

9. Lenné MG, Rudin-Brown CM, Navarro J, Edquist J, Trotter M, Tomasevic N (2011) Driver behaviour at rail level crossings: responses to flashing lights, traffic signals and stop signs in simulated rural driving. Appl Ergon 42:548-554

10. Mcguirl JM, Sarter NB (2006) Supporting trust calibration and the effective use of decision aids by presenting dynamic system confidence information. Hum Factors 48:656-665

11. Railway Industry Safety and Standards Board (2009) Level crossing stocktake

12. Railway Safety Regulators' Panel (2008) Review of national level crossing statistics

13. Rovira E, Cross A, Leitch E, Bonaceto C (2014) Displaying contextual information reduces the costs of imperfect decision automation in rapid retasking of ISR assets. Hum Factors 39(6):1581-1584

14. Seppelt BD, Lee JD (2007) Making adaptive cruise control (ACC) limits visible. Int J Hum Comput Stud 65:192-205

15. Standards Australia (2009) Manual of uniform traffic control devices, Part 7: railway crossings, 3rd edn. Standards Australia, Sydney

16. Tey L-S, Ferreira L, Wallace A (2011) Measuring driver responses at railway level crossings. Accid Anal Prev 43:2134-2141

17. Tey L-S, Kim I, Ferreira L (2012) Evaluating safety at railway level crossings using micro-simulation modelling. In: Transportation research board conference, 2012 Washington DC

18. Wigglesworth EC (1979) The epidemiology of road-rail crossing accidents in Victoria, Australia. J Saf Res 11:162-171

19. Wullems C, Wayth R, Galea V, Nelson-Furnell P (2014) Invehicle railway level crossing warning systems: can intelligent transport systems deliver? In: Conference on railway excellence, Adelaide 